\title{
High-dose hypofractionated X-ray radiotherapy for hepatocellular carcinoma: Tumor responses and toxicities
}

\author{
BING-SHEN HUANG ${ }^{1,2}$, NGAN-MING TSANG ${ }^{1}$, SHI-MING LIN ${ }^{3}$, DENG-YN LIN $^{3}$, JAU-MIN LIEN $^{3}$, \\ CHEN-CHUN LIN ${ }^{3}$, WEI-TING CHEN ${ }^{3}$, WAN-YU CHEN ${ }^{4,5}$ and JI-HONG HONG ${ }^{1}$ \\ ${ }^{1}$ Department of Radiation Oncology, Chang Gung Memorial Hospital and Chang Gung University; \\ ${ }^{2}$ Graduate Institute of Clinical Medicine, Chang Gung University, ${ }^{3}$ Department of Gastroenterology and Hepatology, \\ Chang Gung Memorial Hospital and Chang Gung University, Taoyuan; ${ }^{4}$ Division of Radiation Oncology, \\ Department of Oncology, National Taiwan University Hospital; ${ }^{5}$ Graduate Institute of Clinical Medicine, \\ College of Medicine, National Taiwan University, Taipei, Taiwan
}

Received March 21, 2013; Accepted August 22, 2013

DOI: $10.3892 / \mathrm{ol} .2013 .1582$

\begin{abstract}
Hypofractionated radiotherapy (RT) has been employed to treat hepatocellular carcinoma (HCC). The present study aimed to report the treatment effects, the dose-response associations and the factors that are associated with radiation-induced liver disease (RILD) in a high-dose hypofractionated RT procedure. A total of 40 patients with non-metastatic HCC who underwent RT for local control of irradiated tumors were studied. The treatment technique was that of three-dimensional conformal or intensity-modulated radiation therapy, with a fraction size of $3 \mathrm{~Gy}$ and a total dose of 40-66 Gy in 14-23 fractions. The biologically-effective dose (BED) was 52.0-85.8 $\mathrm{Gy}_{10}$ (median, $74.1 \mathrm{~Gy}_{10}$ ). Tumor regression was observed in 28 patients $(70.0 \%)$ with a complete response, partial response, stable disease and progressive disease status in $11(27.5 \%), 17(42.5 \%)$, five $(12.5 \%)$ and seven patients (17.5\%), respectively. The one-, two- and five-year overall survival (OS) and in-field control (IFC) rates were 60,40 and $21 \%$ and 73, 62 and 56\%, respectively. A positive correlation also emerged between the radiation dose and the IFC ( $\mathrm{P}=0.035)$. Eight of the 40 patients (20\%) developed non-classic RILD. A higher Cancer of the Liver Italian Program score was associated with a higher probability of non-classic RILD ( $\mathrm{P}=0.02)$. The tumor response and IFC rate of HCC following irradiation were significantly dose-dependent. High-dose hypofractionated X-ray RT is a feasible and effective treatment for HCC in patients with good liver function and for those who meet the criteria for a curative attempt.
\end{abstract}

Correspondence to: Dr Ji-Hong Hong, Department of Radiation Oncology, Chang Gung Memorial Hospital and Chang Gung University, No. 5 Fusing Street, Gueishan, Taoyuan 333, Taiwan E-mail: jihong@adm.cgmh.org.tw

Key words: hepatocellular carcinoma, hypofractionated, radiotherapy, toxicities

\section{Introduction}

Hepatocellular carcinoma (HCC) is one of the most common cancers in Asia, where chronic viral hepatitis is common (1). Patients with HCC typically have impaired liver function due to virus- or alcohol-induced cirrhosis or viral hepatitis and only $\sim 20 \%$ are appropriate candidates for surgery (2). The five-year overall survival (OS) rate for patients that are treated by surgery is $30-70 \%$ (3). For those who are not treated with surgery, liver function affected by an underlying liver disease has a strong affect on the clinical outcomes and complicates treatment strategies to a greater extent than for other tumors. Maximal preservation of the normal liver volume and function is a significant consideration in the choice of treatment.

Percutaneous ethanol injection therapy (PEIT) and radiofrequency ablation (RFA) are two major non-surgical local treatments for HCC. PEIT is often used for small HCCs. Higher local failure rates have been identified in patients with tumors of $>3 \mathrm{~cm}$ that have been treated by PEIT, or in those with more than three tumors (4). RFA, which is able to treat tumors of $\leq 5 \mathrm{~cm}$, has a more efficient local control rate than PEIT for small tumors (5). However, RFA is difficult to perform in patients with anatomically unfavorable tumor locations or coagulopathy, as is commonly observed in HCC patients. Transcatheter arterial chemoembolization (TACE), although not considered a curative treatment, is used in patients with poor liver function or those who are not suitable candidates for RFA or PEIT. A systemic review of randomized trials has shown that TACE improves the survival of patients with unresectable HCC (6).

Radiotherapy (RT) has not been widely adopted as a curative treatment modality for HCC due to poor liver tolerance from radiation damage. Improvements in RT techniques, including three-dimensional conformal RT (3DCRT), intensity-modulated RT (IMRT) and image-guided RT (IGRT), provide multiple treatment portals with a reduced volume of liver subjected to high-dose therapy and improved conformity and precision. These techniques increase the prescribed dose and local-control likelihood with acceptable liver toxicity (7). The parallel arrangement of liver-tissue functional subunits 
has facilitated the employment of hypofractionated RT. A large fraction of $\mathrm{HCC}$ was used in proton beam therapy as the normal liver dose may be reduced by its physical characteristic (8). For X-ray, a large fraction size for primary or metastatic liver tumors is provided through stereotactic body RT (9), and clinical trials are being conducted (10).

The present study investigated the use of X-rays with a moderate hypofractionation schema to achieve local control of the irradiated tumor in the treatment of HCC patients. The schema was $3 \mathrm{~Gy} /$ fraction, with a maximal total dose of up to 60-66 Gy if the liver tolerance was acceptable. The total dose of this schema was between the conventional fraction size of 2 Gy and the large fraction size provided by stereotactic body radiosurgery.

\section{Materials and methods}

Patients. The study procedure conformed to the ethical guidelines of the Declaration of Helsinki and approval for the study was obtained from the institution's human research committee (nos. 99-1,924B). Between January 1998 and January 2008, medical records were reviewed for 40 patients with non-metastatic HCC who underwent high-dose RT, with attention to local tumor control. All the patients were treated with 3DCRT or IMRT and were administered a total radiation dose of $>50 \mathrm{~Gy}_{10}$. The patients who were diagnosed with HCC and administered RT of a biologically-effective dose (BED) of $>50 \mathrm{~Gy}_{10}$ using the $\alpha / \beta$ ratio of $10 \mathrm{~Gy}$ were selected for the present study.

Table I provides a summary of the characteristics of the 40 patients, consisting of 10 males and 30 females, with a median age of 63 years (range, $42-82$ years). A total of $32(80.0 \%)$ patients presented with liver cirrhosis (LC) and $15(37.5 \%)$ had a history of esophageal variceal (EV) bleeding. Of the 40 patients, 25 (62.5\%) had Child-Pugh class A (11) LC and 23 patients $(57.5 \%)$ had an Eastern Cooperative Oncology Group (ECOG) performance status of $0-1$. The previous treatments of the 28 patients who were administered RT as a salvage treatment are as follows: Surgery in one patient, TACE in 23, RFA in two, PEIT in 11 and oral chemotherapy in one. The distribution of patients with a Cancer of the Liver Italian program (CLIP) (12) score of 0, 1, 2, 3 and 4 was six, 13, 13, six and two patients, respectively. Portal vein thrombosis (PVT) was present in 13 patients $(32.5 \%)$.

Radiation therapy. The patients were immobilized in a supine position using a vacuum bag with their arms elevated overhead. Contrast-enhanced images were used for target delineation and dynamic computed tomography (CT) was performed as required for tumor identification. The CT images that were captured subsequent to 2007 were obtained by respiration-gating or $4 \mathrm{D}$ techniques. The delineation of the gross tumor volume (GTV) accounted for the organ motion in the 4D CT. The clinical tumor volume (CTV) was obtained by adding a 5-10-mm expansion from the GTV, and the expansion of the planning tumor volume (PTV) was typically $5 \mathrm{~mm}$ for the lateral directions, $0.5-1 \mathrm{~cm}$ for the anterior-posterior direction and $0.5-1.5 \mathrm{~cm}$ for the cephalic-caudal direction. The PTV extension depended on 4D or respiratory-gating CT and whether imaged-guided or respiratory gating was used in
Table I. Patient Characteristics.

Characteristic

Number of patients, n (\%)

Gender

Female

$10(25.0)$

Male

$30(75.0)$

Age, years

$<63$

$20(50.0)$

$\geq 63$

$20(50.0)$

ECOG performance status

0-1

$23(57.5)$

2

17 (42.5)

Liver cirrhosis

No

Yes

$32(80.0)$

EV bleeding history

No

$25(62.5)$

Yes

Child-Pugh class

A

$25(62.5)$

B

15 (37.5)

Previous treatment

No

$12(30.0)$

Yes

$28(70.0)$

Surgery

TACE

$23(82.1)$

RFA

PEIT

$11(39.3)$

$\mathrm{C} / \mathrm{T}$

CLIP score

0

1

$13(32.5)$

$13(32.5)$

$6(15.0)$

$2(5.0)$

Hepatitis

NBNC

$10(25.0)$

B

$6(15.0)$

C

$20(50.0)$

$\mathrm{B}+\mathrm{C}$

$4(10.0)$

Tumor number

Single

$17(42.5)$

Multiple

$23(57.5)$

Tumor size, $\mathrm{cm}$

$<5$

$25(62.5)$

5-10

$14(35.0)$

$1(2.5)$

PVT

No

Yes 
Table I. Continued.

Characteristic

Number of patients, $\mathrm{n}(\%)$

$\begin{array}{ll}\text { AJCC Stage } & \\ \text { I-II } & 21(52.5) \\ \text { III-IV } & 19(47.5)\end{array}$

CLIP, Cancer of the Liver Italian Program; C/T, chemotherapy; ECOG, Eastern Cooperative Oncology Group; EV, esophageal varices; NBNC, non-B/non-C; PEI, percutaneous ethanol injection; PVT, portal vein thrombus; RFA, radiofrequency ablation; TACE, transcatheter arterial chemoembolization; AJCC, American Joint Committee on Cancer.

the treatment. Of the 40 patients, 15 (37.5\%), 7 (17.5\%) and $18(45.0 \%)$ patients underwent 3DCRT, IMRT and 4D planning RT, respectively.

The prescribed dose was defined as a $100 \%$ and $95 \%$, which applied to the CTV and PTV, respectively. The total dose was adjusted by considering the liver tolerance dose with the restriction that $<30 \%$ of normal liver received $>30$ Gy (V30) and the dose restriction was reduced to 27 Gy (V27) for those with Child-Pugh class B disease. The median fraction size was $3 \mathrm{~Gy} /$ fraction and the radiation dose was 40-66 Gy in 14-23 fractions (BED of 52.0-85.8 $\mathrm{Gy}_{10}$ using the $\alpha / \beta$ ratio of $10 \mathrm{~Gy}$; median, $74.1 \mathrm{~Gy}_{10}$ ). The fraction size was reduced to 2-2.5 Gy if the bowel was included in the PTV. The median of the mean liver dose for all the patients was 2,062 cGy (range, $1,008-2,415 \mathrm{cGy})$. The median of $\mathrm{V} 30$ for all the patients was $24 \%$ (range, $12-35 \%$ ).

Follow-up. The patient cases were followed-up at least every three months by CT or ultrasonography during the first year and every six months for up to three years thereafter. The follow-up imaging studies were compared with those that were taken prior to RT and the most significant change in tumor size was regarded as the treatment response. The radiographical tumor response following RT was evaluated using the World Health Organization criteria (13). In-field failure (IFF) was defined as tumor regrowth within the current RT field. Intrahepatic recurrence outside the RT field was defined as an intrahepatic failure (IHF). Distant metastasis (DM) was defined as any recurrence outside the liver.

Classic radiation-induced liver disease (RILD) was defined by the presence of anicteric ascites and the elevation of alkaline phosphatase levels to at least a two-fold increase over the pre-treatment values in the absence of tumor progression. The end-point (occurrence of classic RILD) occurred in patients with good liver function. Non-classic RILD was defined as the elevation of alkaline phosphatase levels to more than five times the upper limit of normal or a decline in liver function (measured by a worsening of the Child-Pugh score by two or more). The end-point was described in patients with poor liver function (virus hepatitis, liver cirrhosis, portal hypertension and Child-Pugh Classes B and C) (14).

Statistics. A univariate cox regression analysis was performed to evaluate the prognostic factors and a multivariate analysis was performed with the forward stepwise procedure using a multiple Cox regression analysis. Survival and IFC were estimated from the first date of RT and the OS rates, and IFC rates were estimated using the Kaplan-Meier method. The Cox regression model was used to investigate the correlation between BED and the IFC. Fisher's exact test and the logistic regression model were also used to evaluate the correlation between the presence of non-classic RILD and the CLIP score. $\mathrm{P}<0.05$ was considered to indicate a statistically significant difference.

\section{Results}

Failure pattern and survival. The failure pattern following a minimum of a two-year follow-up period is shown in Fig. 1. IFF, IHF and DM were observed in 12, 15 and eight patients, respectively, and 10 patients experienced more than one type of recurrence. Following a median follow-up time of 7.7 years for the surviving patients, the one-, two- and five-year OS rates were 60,40 and $21 \%$, respectively (Fig. 2A). OS was significantly affected by the ECOG performance status $(\mathrm{P}=0.012)$, the Child-Pugh classification $(\mathrm{P}=0.003)$, the presence of LC $(P=0.020)$, the CLIP score $(P=0.001)$ and the tumor number $(\mathrm{P}=0.021)$ in the univariate analysis (Table II). The multivariate analysis showed that Child-Pugh classification (Child-Pugh class B vs. A: HR, 5.42; 95\% CI, 2.27-12.95; $\mathrm{P}<0.0001$ ) and the tumor number (multiple vs. single: HR, 4.68; 95\% CI, 2.08-10.53; $\mathrm{P}<0.0001$ ) were the most significant factors affecting OS. The one-, two- and five-year IFC were 72.7, 61.6 and 56.0\%, respectively (Fig. 2B). As shown by the univariate analysis, the factors that were associated with IFC included the tumor number $(\mathrm{P}=0.026)$, treatment response and $\mathrm{BED}\left(\geq 60 \mathrm{~Gy}_{10}\right.$ vs. $<60 \mathrm{~Gy}_{10}, \mathrm{P}=0.021 ; \geq 55 \mathrm{~Gy}_{10}$ vs. $<55 \mathrm{~Gy}_{10}$; $\mathrm{P}=0.001$ ) (Table II). The multivariate analysis revealed that the treatment response (responder vs. non-responder: HR, 0.27; 95\% CI, 0.09-0.83; P=0.023) and BED ( $\geq 55 \mathrm{~Gy}_{10}$ vs. $<55 \mathrm{~Gy}_{10}$ : HR, 0.16; 95\% CI, 0.05-0.55; $\left.\mathrm{P}=0.023\right)$ were the most significant factors for IFC. The one-, two- and five-year intrahepatic control (IHC) and distant-metastasis free survival (DMFS) were 65.4, 56.3 and $41.7 \%$ and 79.8, 75.3 and $75.3 \%$, respectively. No factors associated with IHC were identified. The treatment response alone affected the DMFS $(\mathrm{P}=0.032)$ in the univariate analysis.

Tumor response. Of the 40 patients, 11 (27.5\%) achieved a complete response (CR) following $\mathrm{RT}$ and a partial response (PR) was noted in 17 (42.5\%) patients. The overall response rate was $70.0 \%$. Stable disease (SD) was observed in five patients (12.5\%) and progressive disease (PD) in seven patients (17.5\%) (Table III). A positive correlation trend existed between the radiation dose and the tumor response. A higher BED indicated a higher probability of IFC. Using the Cox regression model, the estimated two-year IFC rates for a BED of $<60 \mathrm{~Gy}_{10}, 60-70 \mathrm{~Gy}_{10}$ and $>70 \mathrm{~Gy}_{10}$ were 43,55 and $70 \%$, respectively $(\mathrm{P}=0.035)$.

Toxicity. Eight of the 40 patients (20\%) were noted to experience a deterioration of the Child-Pugh score by two or more. The median time of non-classic RILD occurrence from RT completion was 39.5 days (range, 15-85 days). Among the 
Table II. Univariate analysis for OS and IFC.

\begin{tabular}{lllll}
\hline & 2-year & & 2-year & \\
Clinical feature & OS, \% & P-value & IFC, \% & P-value \\
\hline
\end{tabular}

\section{Gender}

Female

Male

10.0

50.0

0.151

75.0

Age, years

$\begin{array}{cccc}<63 & 30.0 & & 65.4 \\ \geq 63 & 50.0 & 0.217 & 57.1 \\ \text { ECOG } & & & \\ 0-1 & 52.2 & & 61.5 \\ 2 & 23.5 & 0.012 & 61.8\end{array}$

0.763

Child-Pugh class

$\begin{array}{lllll}\text { A } & 56.0 & & 60.8 & \\ \text { B } & 13.3 & 0.003 & 61.9 & 0.746\end{array}$

Liver Cirrhosis

$\begin{array}{llll}\text { No } & 62.5 & & 72.9 \\ \text { Yes } & 34.4 & 0.02 & 58.2\end{array}$

0.763

EV Bleeding

No

48.0

26.7

0.288

46.3

CLIP Score

$\geq 3$
$<3$

HBV

No

Yes

12.5

46.9

0.019

25.0

76.3

0.034

36.7

57.1

$\begin{array}{lll}50.0 & 0.227 & 75.0\end{array}$

0.312

$\mathrm{HCV}$

No

Yes

31.3

45.8

0.522

39.3

Tumor no.

Single

Multiple

58.8

26.1

0.00

86.9

Tumor size, $\mathrm{cm}$

\begin{tabular}{lrccc}
$<5$ & 48.0 & 0.447 & 74.3 & 0.402 \\
$5-10$ & 28.6 & 0.479 & 39.3 & 0.177 \\
$>10$ & 0.0 & 0.251 & - & - \\
PVT & & & & \\
No & 44.4 & & 66.2 & \\
Yes & 30.8 & 0.286 & 52.9 & 0.704 \\
AJCC stage & & & & \\
I-II & 47.6 & & 74.7 & \\
III-IV & 31.6 & 0.062 & 43.9 & 0.227 \\
Response & & & & \\
Non-responder & 41.7 & & 31.3 & \\
Responder & 39.3 & 0.624 & 74.6 & 0.009 \\
\hline
\end{tabular}

CLIP, Cancer of the Liver Italian Program; ECOG, Eastern Cooperative Oncology Group; EV, esophageal varices; HBV, hepatitis B virus; $\mathrm{HCV}$, hepatitis C virus; IFC, in-field control; OS, overall survival; PVT, portal vein thrombus; AJCC; American Joint Committee on Cancer.
Table III. Tumor response to radiation.

\begin{tabular}{lc}
\hline Response & Number of patients, $(\%)$ \\
\hline CR & $11(27.5)$ \\
PR & $17(42.5)$ \\
SD & $5(12.5)$ \\
PD & $7(17.5)$
\end{tabular}

CR, complete response; PR, partial response; $\mathrm{SD}$, stable disease; $\mathrm{PD}$, progresive disease.

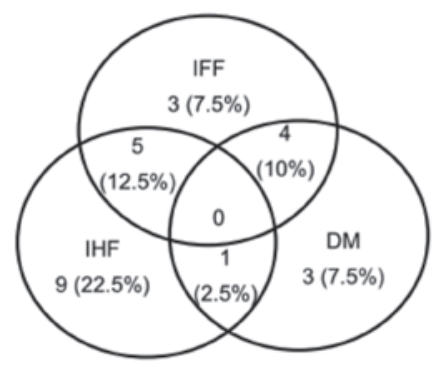

Figure 1. Pattern of failure. The numbers depict cumulative failure sites. IFF, in-field failure; IHF, intrahepatic failure; DM, distant metastasis.

A
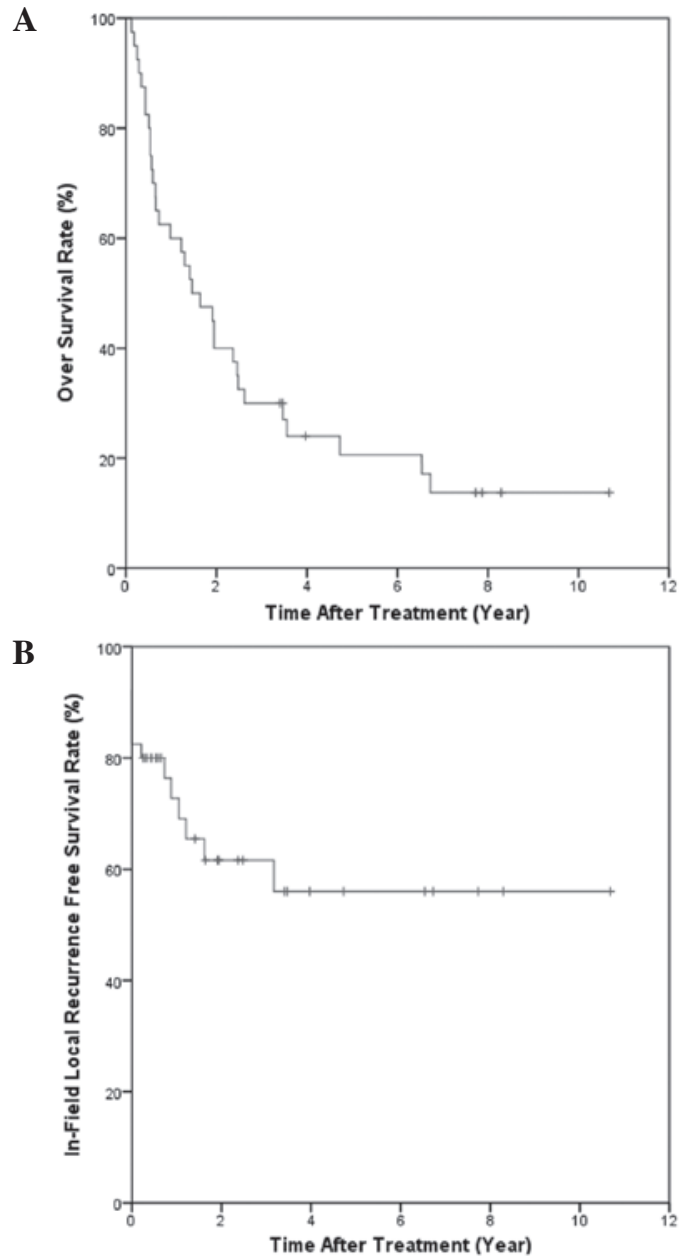

Figure 2. Kaplan-Meier curves of (A) survival and (B) local control. The 1 -, 2- and 5-year OS rates were 60,40 and $21 \%$, respectively. The 1-, 2- and 5 -year local control rates were $72.7,61.6$ and $56.0 \%$, respectively. 
patients who developed non-classic RILD, one (12.5\%), five $(62.5 \%)$ and two (25\%) demonstrated CR, PR and PD, respectively. Six of the eight patients with non-classic RILD exhibited ascites and an increased serum total bilirubin level. Using the logistic regression model, the estimated probability of non-classic RILD for CLIP scores $0,1,2,3$ and 4 were 3, $8.2,21,43.9$ and $69.8 \%$, respectively $(\mathrm{P}=0.02)$. A higher CLIP score was associated with a higher probability of non-classic RILD. A positive association between BED to the tumor and non-classic RILD was not identified by the dose constraints. The probability of non-classic RILD did not increase when the BED to the tumor increased. However, the mean liver dose for the patients who developed RILD was significantly higher than that for the non-RILD patients $(2,322$ cGy vs. $1764 \mathrm{cGy}$; $\mathrm{P}=0.048)$. Classic RILD was not noted in any patients. One patient had a duodenal ulcer confirmed by panendoscopy. The patient who developed the duodenal ulcer underwent 3DCRT with a dose of $54 \mathrm{~Gy}$ in 18 fractions to PTV and the ulcer location was in the $90-95 \%$ isodose region.

\section{Discussion}

RT by X-ray is not routinely used in the curative treatment of HCC. However, HCC has been observed to be more radiosensitive than was previously believed (15). The major limitation has been the poor radiation tolerance of the adjacent normal liver. The first study of the correlation between the dose and complication rate for whole-liver RT was reported by Ingold $e t a l$, who demonstrated that the RILD incidence was 12.5 and $44 \%$ for patients who were treated with 30-35 Gy and $>35$ Gy whole liver RT, respectively (16). In a Radiation Therapy Oncology Group study for liver metastasis, no RILD was observed in patients who were administered 30 Gy whole liver irradiation provided by $1.5 \mathrm{~Gy} / \mathrm{per}$ fraction in two factions per day (17). Lawrence et al (18) revealed that a higher radiation dose (30 Gy whole liver irradiation with a 15 or 30 Gy boost) resulted in a higher tumor response than whole liver RT alone (64\% for the boost group and 39\% for whole liver $\mathrm{RT}$ alone). In addition, it has been shown that the tolerance for liver irradiation may be $35 \mathrm{~Gy}$ for the whole liver, $42 \mathrm{~Gy}$ for $70 \%$ of the liver, 52 Gy for $50 \%$ of the liver and 70 Gy for $30 \%$ of the liver (19).

Dawson et al (20) showed that liver doses associated with a 5\% risk of RILD for uniform irradiation of one-third, two-thirds and the whole liver were 90, 47 and $31 \mathrm{~Gy}$, respectively. Advancements in RT technology have created the possibility of delivering a higher local radiation dose to the liver tumor. A positive correlation between radiation dose and tumor response was observed by Park et al (21). The response rates for doses of $<40,40-50$ and $>50$ Gy were 29, 69 and $77 \%$, respectively. The dose response was established in $<50$ Gy radiation. A PR was observed in $90 \%$ of patients with tumors of $<5 \mathrm{~cm}$, but only in $60 \%$ of those with tumors $>5 \mathrm{~cm}$. Park et al (7) showed that the IFF rate was 46.7 vs. $16.9 \%$ for patients treated with doses of $\leq 50 \mathrm{~Gy}_{10}$ and $>50 \mathrm{~Gy}_{10}$, and concluded that a BED of $50 \mathrm{~Gy}_{10}$ was a criterion for an effective radiation dose. Liu et al (22) showed that an improved OS rate was correlated with the dose delivered to the tumor, particularly for doses of $>50.4 \mathrm{~Gy}$ (1.8 Gy per fraction). Although previous studies have shown that the radiation dose and tumor size affect the treatment results, only a specific dose obtains an enhanced tumor response.

All 40 patients in the present study were administered a greater radiation dose of 52.0-85.8 $\mathrm{Gy}_{10}$ with a median BED of $74.1 \mathrm{~Gy}_{10}$, showing a positive correlation between the radiation dose and tumor response. A higher BED indicated a higher probability of a tumor response. In addition, a BED of $\geq 55 \mathrm{~Gy}_{10}$ was significantly associated with an improved IFC rate. The results show this schema is feasible for HCC curative treatment and that a dose-response correlation exists for tumor control.

The corresponding BED for the hypofractionated RT schema was $52.0-85.8 \mathrm{~Gy}_{10}$ using the $\alpha / \beta$ ratio of $10 \mathrm{~Gy}$ (median, 74.1 $\mathrm{Gy}_{10}$ ). The doses published from 3DCRT with conventional fractionation for HCC were between 33 and 66 Gy (23). A wide range of response (55-92\%), one-year local control (61-78\%) and one-year OS (43-61\%) rates were reported for these doses. Studies have reported similar results to the present treatment strategy with a fraction size of 3-6 Gy/fx, a total dose of 38-68 Gy $(24,25)$, a $55-70 \%$ response rate, a $73-85 \%$ one-year local control rate and a $60-100 \%$ one-year OS rate. Stereotactic body radiotherapy (SBRT) with a diversified fractionation schema showed a $65-100 \%$ one-year local control rate and a $48-93 \%$ one-year OS rate $(9,26)$. However, stringent patient-selection criteria for liver function, tumor location, tumor size and the high-technique demand limit the routine use of SBRT. The present data and data from other published studies have shown that high-dose hypofractionated conformal RT is feasible and yields an improved local control compared with 3DCRT conventional fractionation.

Although higher-dose RT for HCC is achievable with careful patient selection and an improved radiation technique, RILD remains a significant complication. Data from Western countries indicate that a mean liver dose of $28 \mathrm{~Gy}$ in 2-Gy/fx is associated with a 5\% risk of classic RILD, which is characterized by fatigue, weight gain, increased abdominal girth, hepatomegaly, anicteric ascites and an isolated elevation in alkaline phosphatase that is out of proportion with the other liver enzymes (27). For the HCC patients in regions with endemic viral infection, the tolerance dose for RILD was shown to be lower and HBV infection predisposed patients to RILD, particularly for non-classic RILD presenting with jaundice or markedly elevated serum transaminases of more than five times the upper limit of the reference range $(28,29)$. Radiation was shown to induce HBV reactivation, possibly through the bystander effect, and non-classic RILD further complicated the RILD for viral hepatitis-related HCC $(30,31)$.

Eight of the 40 patients in the present study developed non-classic RILD, six of whom had viral hepatitis under the liver-dose constraints of V30<30\%. The most significant prognostic factor for non-classic RILD was a high CLIP score. Previous studies have shown that in addition to a normal liver dose and HBV carrier status, the underlying liver function is also a significant predictive factor for $\operatorname{RILD}(32,33)$. The Child-Pugh classification has often been used to evaluate liver reserve in cirrhosis patients. The present study revealed that the CLIP score, assigning points for the Child-Pugh score, the tumor morphology (solitary, $\leq 50 \%$ of the liver, massive), the serum $\alpha$-fetoprotein level and the presence or absence of 
PVT (12), not only serve as prognostic factors for the survival of HCC patients $(34,35)$, but that they also strongly correlate with RILD incidence.

In the spectrum of local-regional therapy for $\mathrm{HCC}$, the percentage of good surgical candidates for resection is $\sim 20 \%$ and the five-year survival rate following surgery is $\sim 50 \%$. RFA results in five-year survival rates of $50 \%$, with up to $20 \%$ recurrence rates for larger tumors. However, the result is often limited by the size and location of the tumor. For patients with large or multifocal tumors, TACE offers survival benefits compared with the best supportive treatment alone (36-38). However, the five-year survival rate is $<2 \%$ and the recurrence rate is nearly $100 \%$. In the present study, $40 \%$ of patients had Child-Pugh class B liver cirrhosis and $>75 \%$ had viral hepatitis. The tumors in the majority of the patients $(70 \%)$ failed to respond to previous local-regional therapies and were recurrent multiple HCCs of a moderate size. Within this patient population, the strategy resulted in a five-year IFC rate of 56\% and a five-year OS rate of $20.6 \%$. The present study shows that this strategy achieves long-term survival and good local control in certain patients.

In summary, the present study showed that high-dose hypofractionated RT is a feasible and effective treatment for HCC. A positive correlation was identified between the radiation dose and IFC. It was shown that a higher BED indicates a higher probability of IFC. The baseline liver function and the CLIP score should also be evaluated carefully to avoid RILD.

\section{Acknowledgements}

This study was presented as poster in American Society for Radiation Oncology 51th Annual Meeting, Chicago. The authors would like to thank the Statistical Center For Clinical Research, Chang Gung Memorial Hospital, Taoyuan, Taiwan for their assistance with this study.

\section{References}

1. Venook AP, Papandreou C, Furuse J and de Guevara LL: The incidence and epidemiology of hepatocellular carcinoma: a global and regional perspective. Oncologist 15 Suppl 4: 5-13, 2010.

2. Liu CL and Fan ST: Nonresectional therapies for hepatocellular carcinoma. Am J Surg 173: 358-365, 1997.

3. Ye SL, Takayama T, Geschwind J, Marrero JA and Bronowicki JP: Current approaches to the treatment of early hepatocellular carcinoma. Oncologist 15 Suppl 4: 34-41, 2010.

4. Khan KN, Yatsuhashi H, Yamasaki K, et al: Prospective analysis of risk factors for early intrahepatic recurrence of hepatocellular carcinoma following ethanol injection. J Hepatol 32: 269-278, 2000

5. Lin SM, Lin CJ, Lin CC, Hsu CW and Chen YC: Randomised controlled trial comparing percutaneous radiofrequency thermal ablation, percutaneous ethanol injection, and percutaneous acetic acid injection to treat hepatocellular carcinoma of $3 \mathrm{~cm}$ or less. Gut 54: 1151-1156, 2005.

6. Llovet JM and Bruix J: Systematic review of randomized trials for unresectable hepatocellular carcinoma: Chemoembolization improves survival. Hepatology 37: 429-442, 2003.

7. Park W, Lim DH, Paik SW, et al: Local radiotherapy for patients with unresectable hepatocellular carcinoma. Int J Radiat Oncol Biol Phys 61: 1143-1150, 2005.

8. Kawashima M, Furuse J, Nishio T, et al: Phase II study of radiotherapy employing proton beam for hepatocellular carcinoma. J Clin Oncol 23: 1839-1846, 2005.

9. Tse RV, Hawkins M, Lockwood G, et al: Phase I study of individualized stereotactic body radiotherapy for hepatocellular carcinoma and intrahepatic cholangiocarcinoma. J Clin Oncol 26: 657-664, 2008.
10. Toronto UHN: Stereotactic Body Radiation Therapy (SBRT) Hepatocellular Carcinoma. Journal Available from: http://www.clinicaltrials.gov/ct2/show/NCT009143 55 ?term $=$ HCC + SBRT\&rank=00914355 NLM Identifier: NCT00914355. Accessed April 25, 2012.

11. Desmet VJ, Gerber M, Hoofnagle JH, Manns M and Scheuer PJ: Classification of chronic hepatitis: diagnosis, grading and staging. Hepatology 19: 1513-1520, 1994.

12. No authors listed: A new prognostic system for hepatocellular carcinoma: a retrospective study of 435 patients: the Cancer of the Liver Italian Program (CLIP) investigators. Hepatology 28: 751-755, 1998.

13. Miller AB, Hoogstraten B, Staquet M and Winkler A: Reporting results of cancer treatment. Cancer 47: 207-214, 1981.

14. Pan CC, Kavanagh BD, Dawson LA, et al: Radiation-associated liver injury. Int J Radiat Oncol Biol Phys 76: S94-S100, 2010.

15. Cheng SH, Lin YM, Chuang VP, et al: A pilot study of three-dimensional conformal radiotherapy in unresectable hepatocellular carcinoma. J Gastroenterol Hepatol 14: 1025-1033, 1999.

16. Ingold JA, Reed GB, Kaplan HS and Bagshaw MA: Radiation hepatitis. Am J Roentgenol Radium Ther Nucl Med 93: 200-208, 1965.

17. Russell AH, Clyde C, Wasserman TH, Turner SS and Rotman M: Accelerated hyperfractionated hepatic irradiation in the management of patients with liver metastases: results of the RTOG dose escalating protocol. Int J Radiat Oncol Biol Phys 27: 117-123, 1993.

18. Lawrence TS, Dworzanin LM, Walker-Andrews SC, et al: Treatment of cancers involving the liver and porta hepatis with external beam irradiation and intraarterial hepatic fluorodeoxyuridine. Int J Radiat Oncol Biol Phys 20: 555-561, 1991.

19. Lawrence TS, Ten Haken RK, Kessler ML, et al: The use of 3-D dose volume analysis to predict radiation hepatitis. Int J Radiat Oncol Biol Phys 23: 781-788, 1992.

20. Dawson LA, McGinn CJ, Normolle D, et al: Escalated focal liver radiation and concurrent hepatic artery fluorodeoxyuridine for unresectable intrahepatic malignancies. J Clin Oncol 18: 2210-2218, 2000

21. Park HC, Seong J, Han KH, Chon CY, Moon YM and Suh CO: Dose-response relationship in local radiotherapy for hepatocellular carcinoma. Int J Radiat Oncol Biol Phys 54: 150-155, 2002.

22. Liu MT, Li SH, Chu TC, et al: Three-dimensional conformal radiation therapy for unresectable hepatocellular carcinoma patients who had failed with or were unsuited for transcatheter arterial chemoembolization. Jpn J Clin Oncol 34: 532-539, 2004.

23. Seong J, Park HC, Han KH and Chon CY: Clinical results and prognostic factors in radiotherapy for unresectable hepatocellular carcinoma: a retrospective study of 158 patients. Int J Radiat Oncol Biol Phys 55: 329-336, 2003.

24. Liang SX, Zhu XD, Lu HJ, et al: Hypofractionated three-dimensional conformal radiation therapy for primary liver carcinoma. Cancer 103: 2181-2188, 2005

25. Bae SH, Park HC, Lim do H, et al: Salvage treatment with hypofractionated radiotherapy in patients with recurrent small hepatocellularcarcinoma. Int J Radiat Oncol Biol Phys 82: e603-e607, 2012.

26. Kwon JH, Bae SH, Kim JY, et al: Long-term effect of stereotactic body radiation therapy for primary hepatocellular carcinoma ineligible for local ablation therapy or surgical resection. Stereotactic radiotherapy for liver cancer. BMC Cancer 10: 475, 2010.

27. Dawson LA and Ten Haken RK: Partial volume tolerance of the liver to radiation. Semin Radiat Oncol 15: 279-283, 2005.

28. Cheng JC, Wu JK, Lee PC, et al: Biologic susceptibility of hepatocellular carcinoma patients treated with radiotherapy to radiation-induced liver disease. Int J Radiat Oncol Biol Phys 60: 1502-1509, 2004

29. Cheng JC, Liu HS, Wu JK, Chung HW and Jan GJ: Inclusion of biological factors in parallel-architecture normal-tissue complication probability model for radiation-induced liver disease. Int J Radiat Oncol Biol Phys 62: 1150-1156, 2005.

30. Kim JH, Park JW, Kim TH, Koh DW, Lee WJ and Kim CM: Hepatitis B virus reactivation after three-dimensional conformal radiotherapy in patients with hepatitis B virus-related hepatocellular carcinoma. Int J Radiat Oncol Biol Phys 69: 813-819, 2007.

31. Chou CH, Chen PJ, Lee PH, Cheng AL, Hsu HC and Cheng JC: Radiation-induced hepatitis $\mathrm{B}$ virus reactivation in liver mediated by the bystander effect from irradiated endothelial cells. Clin Cancer Res 13: 851-857, 2007. 
32. Liang SX, Zhu XD, Xu ZY, et al: Radiation-induced liver disease in three-dimensional conformal radiation therapy for primary liver carcinoma: the risk factors and hepatic radiation tolerance. Int J Radiat Oncol Biol Phys 65: 426-434, 2006.

33. Xu ZY, Liang SX, Zhu J, et al: Prediction of radiation-induced liver disease by Lyman normal-tissue complication probability model in three-dimensional conformal radiation therapy for primary liver carcinoma. Int J Radiat Oncol Biol Phys 65: 189-195, 2006.

34. Levy I and Sherman M; Liver Cancer Study Group of the University of Toronto: Staging of hepatocellular carcinoma: assessment of the CLIP, Okuda, and Child-Pugh staging systems in a cohort of 257 patients in Toronto. Gut 50: 881-885, 2002.

35. Ueno S, Tanabe G, Sako K, et al: Discrimination value of the new western prognostic system (CLIP score) for hepatocellular carcinoma in 662 Japanese patients. Cancer of the Liver Italian Program. Hepatology 34: 529-534, 2001.
36. Lo CM, Ngan H, Tso WK, et al: Randomized controlled trial of transarterial lipiodol chemoembolization for unresectable hepatocellular carcinoma. Hepatology 35: 1164-1171, 2002.

37. Llovet JM, Real MI, Montana X, et al: Barcelona Liver Cancer Group: Arterial embolisation or chemoembolisation versus symptomatic treatment in patients with unresectable hepatocellular carcinoma: a randomised controlled trial. Lancet 359: 1734-1739, 2002

38. Lin DY, Liaw YF, Lee TY and Lai CM: Hepatic arterial embolization in patients with unresectable hepatocellular carcinoma - a randomized controlled trial. Gastroenterology 94: 453-456, 1988. 